\title{
THE COMPACTIFICABILITY CLASSES: THE BEHAVIOR AT INFINITY
}

\author{
MARTIN MARIA KOVÁR
}

Received 15 October 2004; Revised 18 November 2005; Accepted 28 November 2005

We study the behavior of certain spaces and their compactificability classes at infinity. Among other results we show that every noncompact, locally compact, second countable Hausdorff space $X$ such that each neighborhood of infinity (in the Alexandroff compactification) is uncountable, has $\mathscr{C}(X)=\mathscr{C}(\mathbb{R})$. We also prove some criteria for (non-) comparability of the studied classes of mutual compactificability.

Copyright (c) 2006 Hindawi Publishing Corporation. All rights reserved.

\section{The notation and terminology}

Throughout the paper we mostly use the standard topological notions as in [1] or [2], however with a few exceptions. Space always refers to topological space, usually considered without any additional separation axioms. Especially compactness is understood without the Hausdorff separation axiom. For the terminology related to $\theta$-regularity, we refer the reader to $[3,4]$. An ordinal is the set of smaller ordinals, and a cardinal is an initial ordinal. Let $S$ be a set. The cardinality of $S$ we denote by $|S|$. Let $(X, \tau)$ be a topological space. If we do not wish to specify its topology explicitly, we will sometimes, for our convenience, speak less precisely about the space $X$. Conversely, if we decide to specify the topology of a space $X$ introduced in some previous steps, we will usually denote it by $\tau$ or $\tau_{X}$ (in the case that we will work simultaneously with more topological spaces or more topologies on the same set). In a space $X$, a point $x \in X$ is in the $\theta$ closure of a set $A \subseteq X\left(x \in \operatorname{cl}_{\theta} A\right)$ if every closed neighborhood of $x$ intersects $A$. A filter base $\Phi$ in $X$ has a $\theta$-cluster point $x \in X$ if $x \in \bigcap\left\{\operatorname{cl}_{\theta} F \mid F \in \Phi\right\}$. We say that a space $X$ is $\theta$-regular if every filter base in $X$ with a $\theta$-cluster point has a cluster point. For more detailed characterization of $\theta$-regularity, the reader is referred to [3-5]. The points $x, y$ in a space $X$ are $T_{0}$-separable if there is an open set containing only one of the points $x, y$. The points $x, y$ are $T_{2}$-separable if they have disjoint open neighborhoods. Let $X$ be a space. Two disjoint sets $A, B \subseteq X$ are said to be point-wise separated in $X$ if every $x \in A$, $y \in B$ are $T_{2}$-separable in $X$. Several modifications of local compactness have been defined by various authors in the literature. In this paper, we say that a space is (strongly) locally compact if its every point has a compact (closed) neighborhood. One can easily

Hindawi Publishing Corporation

International Journal of Mathematics and Mathematical Sciences

Volume 2006, Article ID 24370, Pages 1-12

DOI 10.1155/IJMMS/2006/24370 
check that a space is strongly locally compact if and only if it is $\theta$-regular and locally compact. We will often use the following simple, but important property of strongly locally compact spaces. If $X$ is strongly locally compact and $\gamma X$ is a compactification such that $X$ and the remainder $\gamma X \backslash X$ are point-wise separated in $\gamma X$, then $X$ is open in $\gamma X$. A filter in a space $X$ is said to be ultra-closed if it is maximal among all filters in $X$ having a base consisting of closed sets [1]. By the Wallman compactification of $X$, we mean the set $\omega X=X \cup\{y \mid y$ is a nonconvergent ultra-closed filter in $X\}$. The sets $\mathscr{S}(U)=U \cup\{y \mid y \in \omega X \backslash X, U \in y\}$, where $U$ is open in $X$, constitute an open base of $\omega X$. For more detail, we refer the reader to [1]. Some properties of the Wallman compactification of a $\theta$-regular space were studied in [5].

\section{Preliminaries and introduction}

Let us recall some notions and results from the previous papers [6-8]. Let $\left(X, \tau_{X}\right),\left(Y, \tau_{Y}\right)$ be spaces with $X \cap Y=\varnothing$. We say that the space $X$ is compactificable by the space $Y$ or, in other words, $X, Y$ are said to be mutually compactificable if there is a compact topology $\tau_{K}$ on $K=X \cup Y$ such that the topologies on $X, Y$ induced by $\tau_{K}$ coincide with $\tau_{X}, \tau_{Y}$, respectively, and the sets $X, Y$ are point-wise separated in $\left(K, \tau_{K}\right)$. Then we say that the topology $\tau_{K}$ is $\mathscr{b}$-acceptable. Recall that mutually compactificable spaces are always $\theta$-regular, and any two disjoint strongly locally compact spaces are always mutually compactificable [6].

Let Top be the class of all topological spaces. For any pair of two spaces $X, Z$, we define $X \sim Z$ if for every nonempty space $Y$ disjoint from $X, Z$ the space $X$ is compactificable by $Y$ if and only if $Z$ is compactificable by $Y$. It can be easily seen that $\sim$ is reflexive, symmetric, transitive, and hence it is an equivalence relation. Let us denote by $\mathscr{C}(X)$ the equivalence subclass of Top with respect to $\sim$ containing $X$ and call it the compactificability class of $X$. For any spaces $X, Z$, we put $\mathscr{b}(X) \geq \mathscr{C}(Z)$ if for every nonempty space $Y$ it holds: if the space $X$ is compactificable by $Y$ disjoint from $X, Z$, then $Z$ is compactificable by $Y$. The relation $\succeq$ is reflexive, antisymmetric, transitive, and hence it is an order relation between the compactificability classes. If for some spaces $X, Z$ it holds $\mathscr{C}(X) \succeq \mathscr{C}(Z)$ but $\mathscr{C}(X) \neq \mathscr{C}(Z)$, we write $\mathscr{C}(X) \succ \mathscr{C}(Z)$. We proved in [7] that every compactificability class contains a $T_{1}$ representative, but there are compactificability classes with no Hausdorff representatives.

Let $\mathbb{A}=[1, \infty), \mathbb{Q}=[0,1]$ be equipped with the Euclidean topology induced from $\mathbb{R}$, and $\mathbb{D}=\{0,1\}$ equipped with the discrete topology. By $\mathbf{0}$, we denote the constant function equal to 0 . In [8], we proved that for any $k, n \in \mathbb{N}$ the spaces $\mathbb{A}^{k}, \mathbb{R}^{n}, \mathbb{A}^{k} \times \mathbb{R}^{n}, \mathbb{\natural}^{\aleph_{0}} \backslash\{\mathbf{0}\}$, $\mathbb{D}^{N_{0}} \backslash\{\mathbf{0}\}$ are of the same class of mutual compactificability. Also we proved that if $X$ is a noncompact locally connected metrizable generalized continuum, then $\mathscr{C}(X)=\mathscr{C}(\mathbb{R})$. All these spaces are uncountable but second countable, locally compact and Hausdorff. On the other hand, it can be proved that there exist spaces compactificable by $\mathbb{R}$ which are not compactificable by $\mathbb{N}$, but not conversely. Hence, $\mathscr{G}(\mathbb{N}) \succ \mathscr{C}(\mathbb{R})$. Note that we omit the proof now, because it will follow as a corollary from a theorem that will be presented in the next section. Further, it seems that connectedness does not affect the compactificability classes much because $\mathbb{D}^{\mathrm{N}_{0}}$ is homeomorphic to a subspace of $\mathbb{R}$, also known as the Cantor Discontinuum. Hence, there is a natural question whether it is true that every 
uncountable, second countable, or separable locally compact Hausdorff space must be of the same class of compactificability as $\mathbb{R}$. Next we will give a counterexample for this conjecture. After some further investigation we will find out that the essential property which plays the most important role in determining the compactificability classes is the behavior at infinity.

Before we start, let us recapitulate a couple of theorems from the previous papers that we will need in our proofs in the next section. For the proofs of these result, we refer the reader to [7, Theorem 2.12] for Theorem 2.1, and to [8, Theorems 3.1 and 3.2, Corollary 3.2] for Theorems 2.2, 2.3, Corollary 2.1, respectively.

Theorem 2.1. Let $X$ be a $T_{3.5}$ space which is not locally compact and let $Z$ be a strongly locally compact (not necessarily Hausdorff) space. Then $\mathscr{C}(X)$ and $\mathscr{C}(Z)$ are not comparable in the order $\succeq$.

Theorem 2.2. Let $\left(X, \tau_{X}\right)$ be a closed subspace of a strongly locally compact space $\left(Z, \tau_{Z}\right)$. Then, $\mathscr{C}(X) \succeq \mathscr{C}(Z)$.

Recall that by $w(X)$ we denote the weight of a space $(X, \tau)$, that is, the least infinite cardinal $w(X)$, such that $(X, \tau)$ has an open base $\tau_{0} \subseteq \tau$ with $\left|\tau_{0}\right| \leq w(X)$.

Theorem 2.3. Let $(X, \tau)$ be a locally compact Hausdorff space with $w(X)=\mathfrak{m}$, where $\mathfrak{m} \geqslant$ $\aleph_{0}$. Then $\mathscr{C}(X) \succeq \mathscr{C}\left(\mathbb{D}^{\mathfrak{m}} \backslash\{\mathbf{0}\}\right)$.

Corollary 2.4. For any $k, n \in \mathbb{N}$, the spaces $\mathbb{A}^{k}, \mathbb{R}^{n}, \mathbb{A}^{k} \times \mathbb{R}^{n},{\mathbb{\aleph ^ { 0 }}} \backslash\{\mathbf{0}\}, \mathbb{D}^{\mathrm{N}_{0}} \backslash\{\mathbf{0}\}$ are of the same class of mutual compactificability.

\section{Main results}

Our first theorem studies what happens with the classes of mutual compactificability if a closed compact subspace is collapsed to a singleton.

Theorem 3.1. Let $H \subseteq X$ be a compact closed subspace of a topological space $X$. Define an equivalence relation $\sim$ on $X$ by $(x \sim y) \Leftrightarrow[(x=y) \vee(x, y \in H)]$ for any $x, y \in X$. Let $Y=X / \sim$ be the quotient space. Then $\mathscr{C}(X) \succeq \mathscr{C}(Y)$.

Proof. Let $\left(Z, \tau_{Z}\right)$ be a space which is mutually compactificable by $(X, \tau), X \cap Z=\varnothing$. We put $K=X \cup Z$ and denote by $\tau_{K}$ the $\mathscr{C}$-acceptable topology on $K$. We will show that $H$ is closed in $\left(K, \tau_{K}\right)$. Let $y \in K \backslash H$. If $y \in X$, then $y \in X \backslash H \in \tau_{X}$, so there exists $U \in \tau_{K}$ such that $X \backslash H=X \cap U$. Then $U \cap H=\varnothing$, so $y \in U \subseteq K \backslash H$. If $y \in Z$, then from the fact that $X, Z$ are point-wise separated in $\left(K, \tau_{K}\right)$ it follows that there are $U, V \in \tau_{K}$ such that $y \in U, H \subseteq V$, and $U \cap V=\varnothing$. But then $U \cap H=\varnothing$, which implies that $y \in U \subseteq K \backslash H$. Hence, $H$ is closed in $\left(K, \tau_{K}\right)$.

We will extend the equivalence relation $\sim$ to $K$ by setting

$$
(x \sim y) \Longleftrightarrow[(x=y) \text { or }(\{x, y\} \subseteq H)] \text { for every } x, y \in K .
$$

Let $f: K \rightarrow K / \sim$ be the corresponding quotient mapping. We put $L=K_{/ \sim}$ and consider $L$ with its quotient topology $\tau_{L}$. For simplicity, we may identify the singleton equivalence classes with their elements, so the quotient mapping $f: K \backslash H \rightarrow L \backslash\{h\}$ restricted to 
the open set $K \backslash H$ is the identity on $K \backslash H$ and a homeomorphism between the open subspaces $K \backslash H$ and $L \backslash\{h\}$. Since $Z \subseteq K \backslash H=L \backslash\{h\}$, the topology on $Z$ induced from $\left(L, \tau_{L}\right)$ coincides with its original topology $\tau_{Z}$.

Let $V \in \tau_{Y}$. Then $f^{-1}(V) \in \tau_{X}$, so there exists $U \in \tau_{K}$ such that $f^{-1}(V)=U \cap X$. We put $W=(U \cap Z) \cup V$. Clearly, $V=W \cap Y$. Further,

$$
\begin{aligned}
f^{-1}(W) & =f^{-1}(U \cap Z) \cup f^{-1}(V)=(U \cap Z) \cup f^{-1}=(U \cap Z) \cup(U \cap X) \\
& =U \cap(Z \cup X)=U \cap K=U \in \tau_{K} .
\end{aligned}
$$

Then, $W \in \tau_{L}$ because the quotient mapping is continuous. It follows that $\tau_{Y}$ is weaker or equal to the topology on $Y$ induced by $\tau_{L}$. Conversely, let $W \in \tau_{L}$ and denote $V=W \cap Y$. Then $U=f^{-1}(W) \in \tau_{K}$, so $U \cap X \in \tau_{X}$. We have

$$
\begin{aligned}
U \cap X & =f^{-1}(W) \cap X=f^{-1}(W \cap L) \cap X=f^{-1}[W \cap(Z \cup Y)] \cap X \\
& =f^{-1}[(W \cap Z) \cup(W \cap Y)] \cap X=\left[(W \cap Z) \cup f^{-1}(W \cap Y)\right] \cap X \\
& =[W \cap Z \cap X] \cup\left[f^{-1}(V) \cap X\right]=\varnothing \cup f^{-1}(V)=f^{-1}(V) .
\end{aligned}
$$

Let $g: Y \rightarrow X$ be the quotient mapping given by the original (not extended) equivalence $\sim$ on $X$. Then $g$ is a restriction of $f$, so $g^{-1}(V)=f^{-1}(V) \in \tau_{X}$, and by the definition of the quotient topology we have $V \in \tau_{Y}$. Hence, the topology on $Y$ induced from $\left(L, \tau_{L}\right)$ coincides with $\tau_{Y}$.

Let $y \in Y, z \in Z$. We will show that $y, z$ have disjoint open neighborhoods in $\left(L, \tau_{L}\right)$. If $y \neq h$, then $\{y, z\} \subseteq K \backslash H=L \backslash\{h\}$. There exist $U, V \in \tau_{K}$ such that $y \in U, z \in V$, and $U \cap V=\varnothing$. We put $P=U \backslash H, Q=V \backslash H$. Then $P, Q \subseteq K \backslash H=L \backslash\{h\}$, so $P, Q \in \tau_{L}$ and we have $y \in P, z \in Q$, and $P \cap Q=\varnothing$. Let $y=h$. Since $X, Z$ are point-wise separated in $\left(K, \tau_{K}\right)$ and $H$ is compact, there exist $U, V \in \tau_{K}$ such that $H \subseteq U, z \in V$, and $U \cap V=\varnothing$. We put $P=(U \backslash H) \cup\{h\}$. Then

$$
f^{-1}(P)=f^{-1}(U \backslash H) \cup f^{-1}(\{h\})=(U \backslash H) \cup H=U \in \tau_{K} .
$$

Similarly, $f^{-1}(V)=V \in \tau_{K}$. Then $P, V \in \tau_{L}, y=h \in P, z \in V$, and

$$
\begin{aligned}
P \cap V & =[(U \backslash H) \cap V] \cup[\{h\} \cap V]=\varnothing \cup(\{h\} \cap V) \subseteq\{h\} \cap(K \backslash H) \\
& =\{h\} \cap(L \backslash\{h\})=\varnothing .
\end{aligned}
$$

Hence, $Y$ and $Z$ are point-wise separated in $\left(L, \tau_{L}\right)$.

Finally, the compactness of $\left(L, \tau_{L}\right)$ follows from the continuity of the quotient mapping $f: K \rightarrow L$. Now we have a $\mathscr{C}$-acceptable topology on $L=Y \cap Z$, so $\mathscr{C}(X) \succeq \mathscr{C}(Y)$.

Corollary 3.2. Let $X=\mathbb{N} \cup[-1,0]$ with the Euclidean topology induced from $\mathbb{R}$. Then $\mathscr{C}(X)=\mathscr{C}(\mathbb{N})$.

Proof. Clearly, $[-1,0]$ is the closed compact subspace of $X$, so by the previous theorem we have $\mathscr{C}(X) \succeq \mathscr{C}(\mathbb{N})$. The converse inequality follows from the fact that $\mathbb{N}$ is also a closed subspace of $X$, which is strongly locally compact, and from Theorem 2.2. 
Now we will study the behavior of spaces at infinity and its influence on the compactificability classes. For that purpose, we will need some auxiliary assertions. The following lemma is a variation on Cantor-Bendixson theorem (cf., [2, Problem 1.7.10-11, page 59]).

Lemma 3.3. Let $(X, \tau)$ be a second countable space and let $M \subseteq X$ be an uncountable set. Then there is a closed set $C \subseteq X$ such that $M \backslash C$ is (at most) countable and for every open set $O \subseteq X$, either $C \cap O=\varnothing$ or $M \cap O$ is uncountable.

Proof. Let $\sigma \subseteq \tau$ be a countable base for the topology $\tau$. First, it is easy to see that if the condition stated in the theorem holds for every open basic set $O \in \sigma$, then it holds also for every open set from $\tau$. Therefore, we may restrict our considerations to $\sigma$ instead of $\tau$. By transfinite induction, for some ordinal $\delta$, we define a family $\left\{O_{\alpha} \mid \alpha<\delta\right\} \subseteq \sigma$ as follows:

(1) if for every $O \in \sigma$ either $M \cap O$ is empty or uncountable, then $C=\operatorname{cl}_{X} M$ has all the required properties and we are done. Otherwise, there exists $O_{1} \in \sigma$ such that $\mathrm{O}_{1} \cap M$ is nonempty and countable,

(2) now, suppose that we have already chosen $\left\{O_{\beta} \mid \beta<\alpha\right\}$ for some ordinal $\alpha$. If there exists a basic open set $O \in \sigma$ such that $O \cap\left(M \backslash \bigcup_{\beta<\alpha} O_{\beta}\right)$ is nonempty and countable, we let $O_{\alpha}$ be any such set $O$. Otherwise, we stop and put $\delta=\alpha$.

Having the family $\left\{O_{\alpha} \mid \alpha<\delta\right\}$, we put $C=\operatorname{cl}_{X}\left(M \backslash \bigcup_{\alpha<\delta} O_{\alpha}\right)$. Since $\left\{O_{\alpha} \mid \alpha<\delta\right\} \subseteq \sigma$, the ordinal $\delta$ is countable. We have

$$
M=\left(M \backslash \bigcup_{\beta<\alpha} O_{\beta}\right) \cup\left(M \cap \bigcup_{\beta<\alpha} O_{\beta}\right)
$$

and so

$$
\begin{aligned}
O_{\alpha} \cap M & =\left[O_{\alpha} \cap\left(M \backslash \bigcup_{\beta<\alpha} O_{\beta}\right)\right] \cup\left[O_{\alpha} \cap M \cap \bigcup_{\beta<\alpha} O_{\beta}\right] \\
& \subseteq\left[O_{\alpha} \cap\left(M \backslash \bigcup_{\beta<\alpha} O_{\beta}\right)\right] \cup \underset{\beta<\alpha}{\bigcup}\left(O_{\beta} \cap M\right) .
\end{aligned}
$$

The set $O_{1} \cap M$ is countable, from the previous steps $(\boldsymbol{\beta})$ where $\beta<\alpha$, we suppose $O_{\beta} \cap M$ countable and from the current step $(\boldsymbol{\alpha})$ we know that $O_{\alpha} \cap\left(M \backslash \cup_{\beta<\alpha} O_{\beta}\right)$ is countable. Hence, by transfinite induction, $O_{\alpha} \cap M$ is countable as a countable union of countable sets. Then the set

$$
M \backslash C=M \backslash \mathrm{cl}_{X}\left(M \backslash \bigcup_{\alpha<\delta} O_{\alpha}\right) \subseteq M \cap\left(\bigcup_{\alpha<\delta} O_{\alpha}\right)=\bigcup_{\alpha<\delta}\left(O_{\alpha} \cap M\right)
$$

is also countable. Suppose that $C \cap O \neq \varnothing$ for some $O \in \sigma$. Then, also $O \cap\left(M \backslash \bigcup_{\alpha<\delta} O_{\alpha}\right) \neq$ $\varnothing$ and from the last induction step $(\boldsymbol{\delta})$ it follows that $O \cap\left(M \backslash \bigcup_{\alpha<\delta} O_{\alpha}\right)$ is uncountable, because otherwise we could set $O_{\delta}=O$, which would contradict to the fact that $(\boldsymbol{\delta})$ is the last step of the induction. But then also the set $M \cap O$ is uncountable and we can see that $C$ has all the required properties. 
The next lemma is a variation on Cantor's well-known result that $\mathbb{D}^{\aleph_{0}}$ embeds into every nonempty perfect set of reals (cf., [2, Problem 3.12.11, page 230; Problem 4.5.5, page 290]).

Lemma 3.4. Let $(X, \tau)$ be a locally compact, noncompact, second countable Hausdorff space, $\alpha X=X \cup\{\infty\}$ the Alexandroff compactification of $X$. If every neighborhood of $\infty$ is uncountable, then there exists an embedding $e: \mathbb{D}^{\alpha_{0}} \rightarrow \alpha X$ which maps $\mathbf{0}$ to $\infty$.

Proof. By Lemma 3.3, there is a closed set $C \subseteq \alpha X$ such that every open set that intersects $C$ is an uncountable set and such that $X \backslash C$ is countable. Clearly, $\infty \in C$. Thus, without loss of generality we may assume that $C=\alpha X$. In other words, we may assume that every nonempty open subset of $\alpha X$ is uncountable. In particular, it means that no point in $\alpha X$ is isolated.

Since $\alpha X$ is second countable, it is metrizable. Let $d: \alpha X \times \alpha X \rightarrow \mathbb{R}$ be a metric on $X$, which induces the topology $\tau$. For a point $x \in X$ and a positive real number $r>0$, we denote

$$
B(x, r)=\{y \mid y \in X, d(x, y)<r\}
$$

Let $\mathbb{D}^{<\omega}=\bigcup_{n \in \omega} \mathbb{D}^{n}$ be the set of all nonempty finite sequences whose members consist of 0 's and 1's. Every sequence $s \in \mathbb{D}^{<\omega}$ of the length $n$ may be extended by 0 or 1 , respectively, to the sequence having the length $n+1$. We denote the extended sequence by $s^{-} 0$ or $s^{-} 1$, respectively. For every $s \in \mathbb{D}^{<\omega}$, we define inductively an open set $O_{s} \in \tau$ as follows:

(1) we put $x_{0}=\infty$, and as $x_{1}$ we take any point from $X$. We also put $r_{0}=r_{1}=$ $(1 / 3) d\left(x_{0}, x_{1}\right), O_{0}=B\left(x_{0}, r_{0}\right), O_{1}=B\left(x_{1}, r_{1}\right)$.

(2) suppose that $O_{s}=B\left(x_{s}, r_{s}\right)$ is defined for every $s \in \mathbb{D}^{n}$. Let $s \in \mathbb{D}^{n}$. The point $x_{s}$ is not isolated, so there exists $x_{s^{-} 1} \in B\left(x_{s}, 2 / 3 r_{s}\right), x_{s^{\wedge} 1} \neq x_{s}$. Further, we put $x_{s^{-} 0}=x_{s}$, $r_{s^{-} 0}=r_{s^{-} 1}=1 / 3 d\left(x_{s^{-} 0}, x_{s^{-} 1}\right), O_{s^{-} 0}=B\left(x_{s^{-} 0}, r_{s^{-} 0}\right), O_{s^{-} 1}=B\left(x_{s^{-} 1}, r_{s^{-1}}\right)$.

Let $p \in \mathbb{D}^{N_{0}}$. We denote $p_{n}$ the restriction of the infinite sequence $p$ to the first $n$ elements. From the construction it follows that

$$
O_{p_{1}} \supseteq \mathrm{cl}_{\alpha X} O_{p_{2}} \supseteq O_{p_{2}} \supseteq \mathrm{cl}_{\alpha X} O_{p_{3}} \supseteq O_{p_{3}} \supseteq \cdots
$$

Since the space $\alpha X$ is compact, the intersection $\bigcap_{n \in \mathbb{N}} \mathrm{cl}_{\alpha X} O_{p_{n}}$ of the closed balls is nonempty and since $\lim _{n \rightarrow \infty} r_{p_{n}}=0$, it contains exactly one element, say $e(p)$. In particular, $e(\mathbf{0})=\infty$. The mapping $e: \mathbb{D}^{N_{0}} \rightarrow X$ is an injection. Indeed, let $p, q \in \mathbb{D}^{N_{0}}, p \neq q$, and let $n \in \mathbb{N}$ be the least number for which $p_{n} \neq q_{n}$. We have $e(p) \in \operatorname{cl}_{\alpha X} O_{p_{n}}, e(q) \in \operatorname{cl}_{\alpha X} O_{q_{n}}$, but $\mathrm{cl}_{\alpha X} O_{p_{n}} \cap \mathrm{cl}_{\alpha X} O_{q_{n}}=\varnothing$. Thus $e(p) \neq e(q)$. Finally, we will show that $e: \mathbb{D}^{\alpha_{0}} \rightarrow X$ is continuous. Let $O \in \tau$ be an open set containing $e(p)$. There is some $m \in \mathbb{N}$ such that $e(p) \in O_{p_{m}} \subseteq O$. The set $U=\left\{y \mid y \in \mathbb{D}^{\alpha_{0}}, y_{m}=p_{m}\right\}$ is open in $\mathbb{D}^{\alpha_{0}}$ since it is an intersection of $m$ sub-basic sets of the product topology on $\mathbb{D}^{{ }^{N_{0}}}$. But if $y \in U$, then $e(y) \in \mathrm{cl}_{\alpha X} O_{y_{m+1}} \subseteq O_{y_{m}}=O_{p_{m}} \subseteq O$. Hence, $e: \mathbb{D}^{\kappa_{0}} \rightarrow X$ is continuous. Since $\mathbb{D}^{\mathrm{N}_{0}}$ is compact, $e$ is a homeomorphism onto its image.

Finally, we can formulate and prove the main theorem. 
Theorem 3.5. Let $X$ be a locally compact, noncompact, second countable Hausdorff space. If every neighborhood of $\infty \in \alpha X$ contains uncountably many elements of $X$, then $\mathscr{C}(X)=$ $\mathscr{C}(\mathbb{R})$.

Proof. By Lemma 3.4, $X$ contains a closed subspace homeomorphic to $\mathbb{D}^{\mathrm{N}_{0}} \backslash\{\mathbf{0}\}$. Then from Theorems 2.2, 2.3, and Corollary 2.4, we get

$$
\mathscr{C}\left(\mathbb{D}^{\kappa_{0}} \backslash\{\mathbf{0}\}\right) \succeq \mathscr{C}(X) \succeq \mathscr{C}\left(\mathbb{D}^{\aleph_{0}} \backslash\{\mathbf{0}\}\right)=\mathscr{C}(\mathbb{R}),
$$

which consequently gives $\mathscr{C}(X)=\mathscr{C}(\mathbb{R})$. The proof is complete.

So far, our effort was concentrated especially on the problem how to prove that the compactificability classes of two or more different spaces coincide. Perhaps it is just the right time to find some general conditions under which the compactificability classes of different spaces must differ.

Let $\mathfrak{T}$ be some class of directed sets with $\varnothing \notin \mathfrak{T}$. Define the following properties of a topological space $X$ :

(i) property $\alpha(\mathfrak{T})$ : there exists an injective net $\varphi(A, \geq)$ with $A \in \mathfrak{T}, \varphi(A) \subseteq X$, converging in $X$,

(ii) property $\bar{\alpha}(\mathfrak{T})$ : no injective net $\varphi(A, \geq)$ with $A \in \mathfrak{T}, \varphi(A) \subseteq X$, has a cluster point in $X$.

Lemma 3.6. Let $\left(X, \tau_{X}\right)$ be a $T_{1}$ space, $\square=[0,1], K=\omega X \times \square, Y=K \backslash(X \times\{0\})$. We consider $\square$ with its natural, Euclidean topology. Let $\tau_{K}$ be the product topology of the Wallman compactification $\omega X$ of $\left(X, \tau_{X}\right)$ with the Euclidean topology of $\square$ and let $\tau_{Y}$ be the subspace topology on $Y$ induced from $\left(K, \tau_{K}\right)$. Let $a_{1}, a_{2}, \ldots, b_{1}, b_{2}, \ldots$ be two disjoint sequences in $X$ having no cluster point in $X$. Let $\left(Z, \tau_{Z}\right)$ be a topological space disjoint from $Y$ and let $L=Y \cup Z$ be equipped with a topology $\tau_{L}$ such that $\left(Y, \tau_{Y}\right),\left(Z, \tau_{Z}\right)$ are subspaces of $\left(L, \tau_{L}\right)$. Let $c_{1}, c_{2}, \ldots$ be a sequence in $Z$ such that each $c_{i}$ is a cluster point of the nets $\alpha_{i}(t)=\left(a_{i}, t\right)$, $\beta_{i}(t)=\left(b_{i}, t\right)$ with their values in $Y$ for $t \rightarrow 0$. Then the topology $\tau_{L}$ is not $\mathscr{C}$-acceptable.

Proof. Suppose that all the conditions stated in the lemma are satisfied and the topology $\tau_{L}$ is $\mathscr{C}$-acceptable. We put $F=\left\{a_{1}, a_{2}, \ldots\right\}, F=\left\{b_{1}, b_{2}, \ldots\right\}$. Then $F \cap G=\varnothing$ and $F, G$ are closed in $\left(X, \tau_{X}\right)$. Denote $V=X \backslash G \in \tau_{X}, \mathscr{S}(V)=V \cup\{h \mid h \in \omega X \backslash X, V \in h\}$, where we identify the elements of $\omega X \backslash X$ with the nonconvergent ultra-closed filters in $\left(X, \tau_{X}\right)$. The set $\mathscr{S}(V)$ is open in $\omega X$. Let $h \in \omega X \backslash X$ be a cluster point of the sequence $a_{1}, a_{2}, \ldots$ Then $F \in h$, because otherwise, $\mathscr{S}(X \backslash F)$ would be a neighborhood of $h$, which does not contain any element from $a_{1}, a_{2}, \ldots$. Then, $V \in h$ because $F \subseteq X \backslash G=V$. Then $h \in \mathscr{Y}(V)$. Consequently, all cluster points of the sequence $a_{1}, a_{2}, \ldots$ are in $\mathscr{S}(V)$. Analogously, if $g$ is a cluster point of the sequence $b_{1}, b_{2}, \ldots$, then necessarily $G \in g$. Then $V=X \backslash G \notin g$, which gives $g \notin \mathscr{S}(V)$. Therefore, all cluster points of the sequence $b_{1}, b_{2}, \ldots$ are outside of $\mathscr{Y}(V)$, that is, in $\omega X \backslash \mathscr{Y}(V)$. Then both sequences $a_{1}, a_{2}, \ldots, b_{1}, b_{2}, \ldots$ have no common cluster point in $\omega X$.

Denote $M=\mathbb{N} \times(0,1]$. For every $(k, t),(l, s) \in M$, we define

$$
(k, t) \leq(l, s) \Longleftrightarrow k \geq l, \quad t \leq s .
$$


Further, for every $(k, t) \in M$, we put

$$
\varphi(k, t)=\alpha_{k}(t)=\left(a_{k}, t\right), \quad \chi(k, t)=\beta_{k}(t)=\left(b_{k}, t\right) .
$$

The nets $\varphi(M, \geq), \chi(M, \geq)$ have their values in $Y$ and they have no cluster point in $X \times$ $\{0\}$. Suppose that, for instance, $\varphi(M, \geq)$ has a cluster point $z \in Z$. Then there exists a net $\varphi^{\prime}\left(M^{\prime}, \sqsupseteq\right)$ finer than $\varphi(M, \geq)$, which converges to $z$. Since $K$ is compact, $\varphi^{\prime}\left(M^{\prime}, \sqsupseteq\right)$ has a cluster point, say $y \in K$. But $y$ is also a cluster point of $\varphi(M, \geqslant)$, which has no cluster point in $X \times\{0\}$, so $y \in K \backslash(X \times\{0\})=Y$. However, since $\tau_{L}$ is $\mathscr{C}$-acceptable, $Y$ and $Z$ are point-wise separated in $\left(L, \tau_{L}\right)$. This is not possible since $\varphi^{\prime}\left(M^{\prime}, \sqsupseteq\right)$ converges to $z \in Z$ and has a cluster point $y \in Y$, both in the topology $\tau_{L}$, which on $Y$ coincides with the topology induced from $\left(K, \tau_{K}\right)$. Hence, the net $\varphi(M, \geq)$, and similarly also $\chi(M, \geq)$, has no cluster point in $Z$.

Let $w \in L$ be the cluster point of the sequence $c_{1}, c_{2}, \ldots$. Take $W \in \tau_{L}$ such that $w \in W$. Let $\left(k_{0}, t_{0}\right) \in M$. There exists $k \geq k_{0}$ such that $c_{k} \in W$. However, $c_{k}$ is a cluster point of the nets $\alpha_{k}(t), \beta_{k}(t)$ for $t \rightarrow 0$. So, there exist $t, s \in(0,1], t \leq t_{0}, s \leq t_{0}$ such that $\alpha_{k}(t)=$ $\left(a_{k}, t\right)=\varphi(k, t) \in W$ and $\beta_{k}(s)=\left(b_{k}, s\right)=\chi(k, s) \in W$. We have $(k, t) \geq\left(k_{0}, t_{0}\right)$ and $(k, s) \geq$ $\left(k_{0}, t_{0}\right)$, which mean that $w \in L$ is a common cluster point of the nets $\varphi(M, \geq), \chi(M, \geq)$. Because of the previous paragraph $w \notin Z$, so $w \in Y=K \backslash(X \times\{0\})=(\omega X \times(0,1]) \cup$ $[(\omega X \backslash X) \times\{0\}]$. However, it is not possible that $w \in \omega X \times(0,1]$, because in this case $w \in \omega X \times(\varepsilon, 1]$ for sufficiently small $\varepsilon>0$. The set $\omega X \times(\varepsilon, 1]$ is open in $\tau_{Y}$, but it does not contain $\varphi(k, t)$ or $\chi(k, t)$ if $(k, t) \geq(1, \varepsilon)$. Hence, it remains $w \in(\omega X \backslash X) \times\{0\}$. Then $w=(v, 0)$, where $v \in \omega X \backslash X$. But this implies that $v$ is a common cluster point of the sequences $a_{1}, a_{2}, \ldots, b_{1}, b_{2}, \ldots$, which is a contradiction.

Thus our assumption that $\tau_{L}$ is $\mathscr{b}$-acceptable is incorrect.

Theorem 3.7. Let $\left(X, \tau_{X}\right)$ be a $\theta$-regular $T_{1}$ space containing a discrete infinite sequence of subspaces $P_{1}, P_{2}, \ldots$ with the property $\alpha(\mathfrak{T})$. Then for any space $\left(Z, \tau_{Z}\right)$ with the property $\bar{\alpha}(\mathfrak{T})$ it follows $\mathscr{C}(X) \nsucceq \mathscr{C}(Z)$.

Proof. Suppose $\mathscr{C}(X) \succeq \mathscr{C}(Z)$. We put $\mathbb{\square}=[0,1]$ with the Euclidean topology, $K=\omega X \times$ ], $Y=K \backslash(X \times\{0\})$. We consider the product topology $\tau_{K}$ on $K$ and the topology $\tau_{Y}$ induced from $\left(K, \tau_{K}\right)$ on $Y$. Clearly, the spaces $\left(X, \tau_{X}\right)$ and $X \times\{0\}$ with the topology induced from $\left(K, \tau_{K}\right)$ are homeomorphic. Since $\left(X, \tau_{X}\right)$ is $\theta$-regular, $\omega X \backslash X$ and $X$ are pairwise separated in $\omega X$. Then, also $X \times\{0\}$ and $Y$ are pairwise separated in $\left(K, \tau_{K}\right)$. Then $\mathscr{C}(X \times\{0\})=\mathscr{C}(X) \geq \mathscr{C}(Z)$. Hence, there exists a $\mathscr{C}$-acceptable topology $\tau_{L}$ on $L=$ $Y \cap Z$, where $Z$ is supposed to be disjoint from $Y$ (if $Y \cap Z \neq \varnothing$, we will replace $Z$ by a homeomorphic copy, disjoint from $Y)$.

Let $\xi_{i}\left(A_{i}, \geq\right)$ be an injective net in $P_{i}$ such that $A_{i} \in \mathfrak{T}$, having a limit $l_{i} \in P_{i}$. For any $\alpha \in A_{i}$ and $t \in(0,1]$, we define

$$
\zeta_{i, \alpha}(t)=\left(\xi_{i}(\alpha), t\right) \in P_{i} \times(0,1] \subseteq X \times(0,1] \subseteq Y .
$$

If we consider $(0,1]$ as a directed set, then $\zeta_{i, \alpha}$ is a net with values in $Y$ and a limit $\left(\xi_{i}(\alpha), 0\right) \in X \times\{0\}$ for $t \rightarrow 0$. Since the net $\zeta_{i, \alpha}$ has a limit in $X \times\{0\}$ and the sets $X \times\{0\}$, 
$Y$ are pointwise separated in $\left(K, \tau_{K}\right), \zeta_{i, \alpha}$ has no cluster point in $Y$. Since $\left(L, \tau_{L}\right)$ is compact, $\zeta_{i, \alpha}$ has a cluster point in $Z$, say $\psi_{i}(\alpha) \in Z$. Then $\psi_{i}\left(A_{i}, \geq\right)$ is a net in $Z$, having $A_{i} \in \mathfrak{T}$.

Suppose that $\psi_{i}$ is injective. Since $\left(Z, \tau_{Z}\right)$ has the property $\bar{\alpha}(\mathfrak{T})$, the net $\psi_{i}\left(A_{i}, \geq\right)$ has no cluster point in $Z$, so it must have a cluster point in $Y$, say $y \in Y$. We denote $M_{i}=$ $A_{i} \times(0,1]$ and for every $(\alpha, t),(\beta, s) \in M_{i}$, we define

$$
\begin{gathered}
(\alpha, t) \sqsupseteq(\beta, s) \Longleftrightarrow \alpha \geq \beta, t \leq s^{\prime}, \\
\varphi_{i}(\alpha, t)=\zeta_{i, \alpha}(t)=\left(\xi_{i}(\alpha), t\right) .
\end{gathered}
$$

For every $i \in \mathbb{N}$, we have a net $\varphi_{i}\left(M_{i}, \sqsupseteq\right)$ with values in $Y$. Let us show that $\varphi_{i}\left(M_{i}, \sqsupseteq\right)$ has the limit $\left(l_{i}, 0\right) \in X \times\{0\}$ in $\left(K, \tau_{K}\right)$. Choose $V \in \tau_{X}$ such that $l_{i} \in V$ and $\varepsilon>0$. Then there exists $\alpha_{0} \in A_{i}$ such that $\xi_{i}(\alpha) \in V$ for $\alpha \geqslant \alpha_{0}$. Then, for every $(\alpha, t) \in M_{i}$ such that $\left(\alpha, t_{i}\right) \sqsupseteq\left(\alpha_{0}, \varepsilon / 2\right)$, we have $\varphi_{i}(\alpha, t)=\left(\xi_{i}(\alpha), t\right) \in V \times(0, \varepsilon)$. Then, $\varphi_{i}\left(M_{i}, \sqsupseteq\right)$ converges to $\left(l_{i}, 0\right) \in X \times\{0\}$. Let $W \in \tau_{Y}$ be an open neighborhood of $y \in Y$. There exists $U \in \tau_{L}$ such that $W=Y \cap U$. Let $(\alpha, t) \in M_{i}$. There exists $\beta \in A_{i}, \beta \geq \alpha$ such that $\psi_{i}(\beta) \in U$. But $\psi_{i}(\beta)$ is a cluster point of the net $\zeta_{i, \beta}$. Hence, there exists $s \in(0,1], s \leq t$ such that $\varphi_{i}(\beta, s)=\zeta_{i, \beta}(s)=\left(\xi_{i}(\beta), s\right) \in U$. Since the net $\varphi_{i}\left(M_{i}, \sqsupseteq\right)$ has its values in $Y$, we have $\varphi_{i}(\beta, s) \in W$. Then $y \in Y$ is a cluster point of $\varphi_{i}\left(M_{i}, \sqsupseteq\right)$, which is a contradiction, because in the previous step we proved that $\varphi_{i}\left(M_{i}, \sqsupseteq\right)$ has the limit $\left(l_{i}, 0\right) \in X \times\{0\}$ and the sets $X \times\{0\}, Y$ are point-wise separated in $\left(K, \tau_{K}\right)$. Hence, the only possible conclusion is that $\psi_{i}$ is not injective.

By the previous paragraph, there exist $\alpha_{i}, \beta_{i} \in A_{i}$ such that $\alpha_{i} \neq \beta_{i}$ and $\psi_{i}\left(\alpha_{i}\right)=\psi_{i}\left(\beta_{i}\right)$. We put $a_{i}=\xi_{i}\left(\alpha_{i}\right), b_{i}=\xi_{i}\left(\beta_{i}\right), c_{i}=\psi_{i}\left(\alpha_{i}\right)=\psi_{i}\left(\beta_{i}\right)$. Since the net $\xi_{i}\left(A_{i}, \geq\right)$ is injective, $a_{i} \neq b_{i}$. We have $a_{i}, b_{i} \in P_{i}$, so the sequences $a_{1}, a_{2}, \ldots$ and $b_{1}, b_{2}, \ldots$ are disjoint because of the discreteness of the collection $\left\{P_{1}, P_{2}, \ldots\right\}$. For the same reason, they have no cluster point in $X$. Moreover, for every $i \in \mathbb{N}, c_{i}=\psi_{i}\left(\alpha_{i}\right)=\psi_{i}\left(\beta_{i}\right) \in Z$ is a cluster point of the nets $\zeta_{i, \alpha_{i}}(t)=\left(\xi_{i}\left(\alpha_{i}\right), t\right)=\left(a_{i}, t\right), \zeta_{i, \beta_{i}}(t)=\left(\xi_{i}\left(\beta_{i}\right), t\right)=\left(b_{i}, t\right)$ for $t \rightarrow 0$ in $\left(L, \tau_{L}\right)$. By Lemma 3.6, the topology $\tau_{L}$ is not $\mathscr{C}$-acceptable, which is a contradiction. Therefore, it must be $\mathscr{C}(X) \nsucceq \mathscr{C}(Z)$.

Modifying the technique of the proof of the previous theorem analogously, we can obtain the following result.

Theorem 3.8. Let $\left(X, \tau_{X}\right)$ be a $\theta$-regular $T_{1}$ space containing a discrete infinite sequence of subspaces $P_{1}, P_{2}, \ldots$ with the property $\left|P_{i}\right|>\kappa$ ( $\kappa$ is a cardinal number). Then for any space $\left(Z, \tau_{Z}\right)$ with the property $|Z| \leq \kappa$ it follows $\mathscr{C}(X) \nsucceq \mathscr{C}(Z)$.

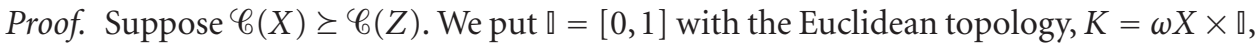
$Y=K \backslash(X \times\{0\})$. We consider the product topology $\tau_{K}$ on $K$ and the topology $\tau_{Y}$ induced from $\left(K, \tau_{K}\right)$ on $Y$. The space $X \times\{0\}$, with the topology induced from $\left(K, \tau_{K}\right)$, is homeomorphic to $\left(X, \tau_{X}\right)$ and pairwise separated from $Y$ in $\left(K, \tau_{K}\right)$. Then $\mathscr{C}(X \times$ $\{0\}) \succeq \mathscr{C}(Z)$. Therefore, there exists a $\mathscr{C}$-acceptable topology $\tau_{L}$ on $L=Y \cap Z$ (where $Z$ is supposed to be disjoint from $Y$ ). 
Let $i \in \mathbb{N}$. For every $p \in P_{i}$, we put $\zeta_{i, p}(t)=(p, t)$. The net $\zeta_{i, p}((0,1], \leq)$ has its values in $Y$ and has the limit $(p, 0) \in X \times\{0\}$ for $t \rightarrow 0$. We put

$$
Q_{i}=\left\{q \mid q \in Z, q \text { is a cluster point of the net } \zeta_{i, p}((0,1], \leq)\right\} \text {. }
$$

Then $\left|Q_{i}\right| \leq \kappa<\left|P_{i}\right|$, so there exist $a_{i}, b_{i} \in P_{i} \subseteq X, a_{i} \neq b_{i}$ such that the nets $\zeta_{i, a_{i}}(t)=$ $\left(a_{i}, t\right), \zeta_{i, b_{i}}(t)=\left(b_{i}, t\right)$ have the same cluster point $c_{i} \in Z$ for $t \rightarrow 0$ in the topology $\tau_{L}$. Since the collection $\left\{P_{1}, P_{2}, \ldots\right\}$ is discrete in $\left(X, \tau_{X}\right)$, the sequences $a_{1}, a_{2}, \ldots$ and $b_{1}, b_{2}, \ldots$ are disjoint and have no cluster point in $X$. By Lemma 3.6, the topology $\tau_{L}$ is not $\mathscr{C}_{-}$ acceptable, which is a contradiction. Hence, $\mathscr{C}(X) \nsucceq \mathscr{C}(Z)$.

The next corollary summarizes some relationships between the compactificability classes of some frequently used and well-known spaces. These properties follow from the theory presented in this paper and in the three previous papers [6-8].

COROLLARY 3.9. The following statements are satisfied:

(i) let $X, Z$ be two infinite discrete spaces with different cardinalities $|X|>|Z|$. Then $\mathscr{C}(Z)>\mathscr{C}(X)$

(ii) $\mathscr{C}(\mathbb{R} \backslash \mathbb{Q}) \nsucceq \mathscr{C}(\mathbb{Q})$,

(iii) $\operatorname{Comp} \succ \mathscr{C}(\mathbb{N}) \succ \mathscr{C}(\mathbb{R})$,

(iv) $\mathscr{C}(\mathbb{Q}), \mathscr{C}(\mathbb{R} \backslash \mathbb{Q})$, and $\mathscr{C}\left((0,1)^{\aleph_{0}}\right)$ are incomparable with Comp, $\mathscr{C}(\mathbb{N}), \mathscr{C}(\mathbb{R})$,

(v) let $X=\{n-1 / m \mid n, m \in \mathbb{N}\}$ be a subspace of $\mathbb{R}$. Then

$$
\mathscr{C}(\mathbb{N}) \succ \mathscr{C}(X) \succ \mathscr{C}(\mathbb{R})
$$

(vi) let $\omega=\aleph_{0}=\mathbb{N}$, and let $\omega_{1}=\aleph_{1}$ be the first uncountable cardinal, both considered as ordinal number, with the interval topology. Then

$$
\mathscr{C}\left(\omega \times\left(\omega_{1}+1\right)\right) \nsucceq \mathscr{C}\left(\omega_{1}\right) .
$$

Proof. (i) Let $|X|>|Z|=\kappa \geq \aleph_{0}$. Then there exists a decomposition of $X$ into countably many pairwise disjoint subsets $P_{i} \subseteq X, i \in \mathbb{N}$, such that, for each $i \in \mathbb{N}$, we have $\left|P_{i}\right|=$ $|X|>\kappa$. From Theorem 3.8 it follows $\mathscr{C}(X) \nsucceq \mathscr{C}(Z)$. Further, by Theorem 2.2 it holds $\mathscr{C}(Z) \succeq \mathscr{C}(X)$. Then $\mathscr{C}(Z) \succ \mathscr{C}(X)$.

(ii) For every $n \in \mathbb{N}$, we put $P_{n}=[2 n, 2 n+1] \cap(\mathbb{R} \backslash \mathbb{Q})$. Then $\left|P_{n}\right|>|\mathbb{Q}|=\aleph_{0}$, and the family $\left\{P_{n} \mid n \in \mathbb{N}\right\}$ is discrete. By Theorem 3.8, we have $\mathscr{C}(\mathbb{R} \backslash \mathbb{Q}) \nsucceq \mathscr{C}(\mathbb{Q})$.

(iii) It is clear that Comp $\succ \mathscr{G}(\mathbb{N})$. We put $P_{n}=[2 n, 2 n+1]$ for every $n \in \mathbb{N}$. We have $\left|P_{n}\right|>|\mathbb{N}|=\mathfrak{N}_{0}$, so by Theorem 3.8 we get $\mathscr{C}(\mathbb{R}) \nsucceq \mathscr{C}(\mathbb{N})$. But $\mathbb{N}$ is a closed subspace of $\mathbb{R}$, so by Theorem 2.2 we have $\mathscr{C}(\mathbb{N}) \geq \mathscr{C}(\mathbb{R})$. Then $\mathscr{C}(\mathbb{N}) \succ \mathscr{C}(\mathbb{R})$.

(iv) The spaces $\mathbb{Q}, \mathbb{R} \backslash \mathbb{Q}$, and $(0,1)^{\aleph_{0}}$ are $T_{3.5}$, but not locally compact. The compact spaces as well as $\mathbb{N}$ or $\mathbb{R}$ are strongly locally compact. The assertion now follows directly from [7, Theorem 2.1].

(v) We take $\mathfrak{T}=\{(\mathbb{N}, \geq)\}$, where we consider the natural, linear order $\geq$ on $\mathbb{N}$. Then any injective net $\varphi(A, \geq)$ with $A \in \mathfrak{T}$ is an injective sequence, which clearly has no cluster point in $\mathbb{N}$. However, if we put $P_{n}=\{n-1 / m \mid m \in \mathbb{N}, m \geq 2\} \cup\{n\}$, then $P_{n} \subseteq$ $X$ contains an injective sequence $n-1 / 2, n-1 / 3, n-1 / 4, \ldots$ converging to $n \in P_{n}$. The 
family $\left\{P_{1}, P_{2}, \ldots\right\}$ is discrete. By Theorem 3.7 it holds $\mathscr{C}(X) \nsucceq \mathscr{C}(\mathbb{N})$. On the other hand, $\mathbb{N}$ is a closed subspace of $X$, so by Theorem $2.2, \mathscr{C}(\mathbb{N}) \succeq \mathscr{C}(X)$. Hence, $\mathscr{C}(\mathbb{N}) \succ \mathscr{C}(X)$. For proving $\mathscr{b}(X) \succ \mathscr{C}(\mathbb{R})$, we may adjust (iii) and use the fact that $X$ is a closed countable subspace of $\mathbb{R}$.

(vi) We take $\mathfrak{T}=\left\{\left(\omega_{1}, \geq\right)\right\}$, where $\geq$ is the order of ordinals. An injective net $\varphi$ $\left(\omega_{1}, \geq\right)$ has no cluster point in $\omega_{1}$, since any uncountable subset of $\omega_{1}$ is not bounded. On the other hand, $P_{n}=\{n\} \times\left(\omega_{1}+1\right)$ clearly contains values of an injective net $\varphi_{n}\left(\omega_{1}, \geq\right)$ given by $\varphi_{n}(\alpha)=(n, \alpha)$, converging to the point $\left(n, \omega_{1}\right) \in P_{n}$. Applying Theorem 3.7, we get $\mathscr{b}\left(\omega \times\left(\omega_{1}+1\right)\right) \nsucceq \mathscr{C}\left(\omega_{1}\right)$.

However, we will note that there are still much more open questions than answers and solutions. Among numerous very natural questions that one certainly may ask, we can only list some of them.

Question 3.10. Is Comp the only class above $\mathscr{C}(\mathbb{N})$ ? Is it true that a space $X$ is compact if and only if $\mathscr{C}(X) \succ \mathscr{C}(\mathbb{N})$ ?

Question 3.11. Which compactificability classes have the representatives among the subspaces of the real line and which are their relationships?

Remark to the previous question that we know almost nothing about the compactificability classes represented by the non-locally compact subspaces of the real line, like $\mathscr{C}(\mathbb{Q})$ or $\mathscr{C}(\mathbb{R} \backslash \mathbb{Q})$. Perhaps some set-theoretic axioms like $\mathrm{CH}$ may affect the next open question.

Question 3.12. Is it true that between $\mathscr{C}(\mathbb{N})$ and $\mathscr{C}(\mathbb{R})$ there are infinitely or even uncountably many compactificability classes?

Question 3.13. Is it true that $\mathscr{b}\left(\omega_{1}\right) \succ \mathscr{C}\left(\omega \times\left(\omega_{1}+1\right)\right)$ ?

Question 3.14. Which relationships are there between the compactificability classes represented by the spaces of ordinals?

Question 3.15. Which properties have the compactificability classes represented by spaces constructed from the classic examples that were given over the years (Sorgenfrey line, Niemytzki plane, ...)?

It is known that each compactificability class has a $T_{1}$ representative, however, there exist classes of mutual compactificability with no Hausdorff representatives. Hence, we will close the paper by the following natural question.

Question 3.16. Is it true that every compactificability class contains a sober or sober $T_{1}$ representative?

\section{Acknowledgment}

The author acknowledges support from Grant no. 201/03/0933 of the Grant Agency of the Czech Republic and from the Research Intention MSM 0021630503 of the Ministry of Education of the Czech Republic. 


\section{The behavior at infinity}

\section{References}

[1] Á. Császár, General Topology, Akademiai Kiadó, Budapest, 1978.

[2] R. Engelking, General Topology, Sigma Series in Pure Mathematics, vol. 6, Heldermann, Berlin, 1989.

[3] D. S. Janković, $\theta$-regular spaces, International Journal of Mathematics and Mathematical Sciences 8 (1985), no. 3, 615-619.

[4] M. M. Kovár, On $\theta$-regular spaces, International Journal of Mathematics and Mathematical Sciences 17 (1994), no. 4, 687-692.

[5] _ A remark on On $\theta$-regular spaces, International Journal of Mathematics and Mathematical Sciences 21 (1998), no. 1, 199-200.

[6] __ Mutually compactificable topological spaces, preprint.

[7] _ The classes of mutual compactificability, to appear in International Journal of Mathematics and Mathematical Sciences.

[8] - The compactificability classes of certain spaces, to appear in International Journal of Mathematics and Mathematical Sciences.

Martin Maria Kovár: Department of Mathematics, Faculty of Electrical Engineering and Communication, University of Technology, Technická 8, Brno 616 69, Czech Republic E-mail address: kovar@feec.vutbr.cz 




Advances in

Operations Research

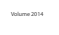

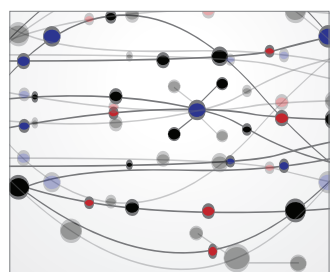

\section{The Scientific} World Journal
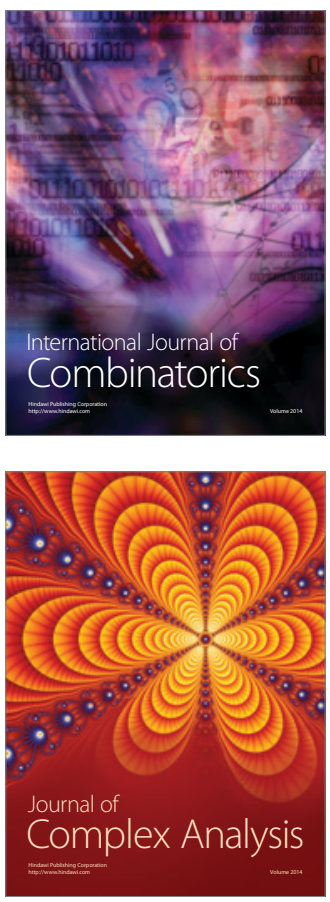

International Journal of

Mathematics and

Mathematical

Sciences


Journal of

Applied Mathematics
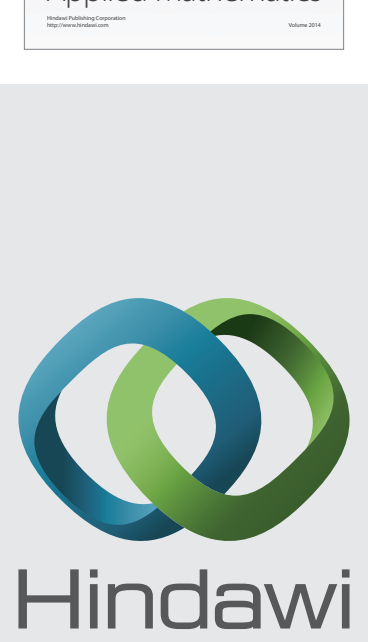

Submit your manuscripts at http://www.hindawi.com


Mathematical Problems in Engineering


Journal of

Function Spaces
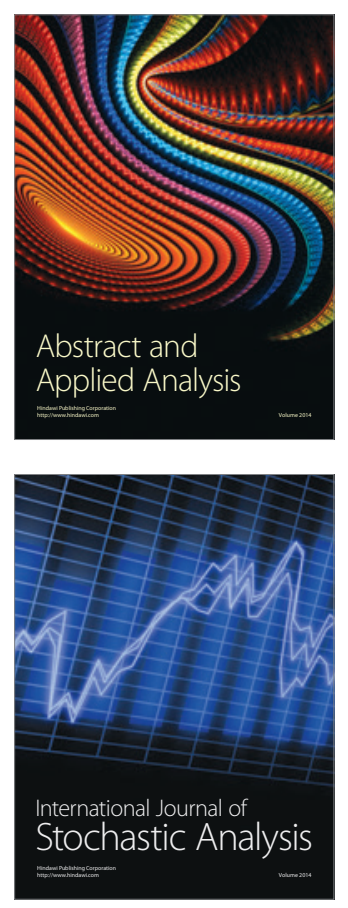

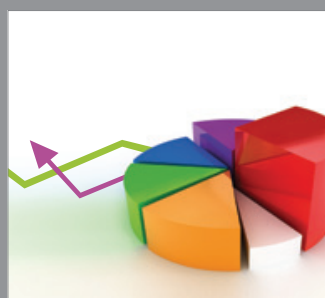

ournal of

Probability and Statistics

Promensencen
